\title{
PARALLEL ALGORITHMS FOR FE-BE COUPLING
}

\author{
R. Perera and E. Alarcon \\ Department of Structural Mechanics, Technical University of Madrid, Madrid, Spain
}

\begin{abstract}
When non linear physical systems of infinite extent are modelled, such as tunnels and perforations, it is necessary to simulate suitably the solution in the infinite as well as the non linearity. The finite element method (FEM) is a well known procedure for simulating the non linear behavior. However, the treatment of the infinite field with domain truncations is often questionable. On the other hand, the boundary element method (BEM) is suitable to simulate the infinite behavior without truncations. Because of this, by the combination of both methods, suitable use of the advantages of each one may be obtained. Several possibilities of FEMBEM coupling and their performance in some practical cases are discussed in this paper. Parallelizable coupling algorithms based on domain decomposition are developed and compared with the most traditional coupling methods.
\end{abstract}

\section{Introduction}

Coupled finite element and boundary element methods are especially well-suited for dealing with non linear problems in an infinite halfspace. The boundary element method (BEM) represents an ideal approach for treating the infinite and semiinfinite domains involved. The finite element method (FEM), on the other hand, is capable of dealing with the non-homogeneity and non-linearity of the domains. A coupling method combining boundary and finite elements appears to be very attractive since it exploits the best features of each method and simultaneously minimizes their weak points. The general technique of FE-BE coupling was developed by Zienkiewicz et al [1].

Finite element and boundary element methods lead to very different kinds of systems: systems with sparse symmetric positive definite matrices for FEM [2] and systems with full non symmetric matrices for collocation BEM [3]. The first kind of systems may be efficiently solved by iterative methods while the second kind is solved by direct methods. When general coupling is performed the result is a large matrix partially sparsed but with full non symmetric blocks and neither a direct method nor an iterative method is convenient to solve such a system.

On the other hand, with the advent of modern multiprocessor computers, new approaches $[4,5,6]$ have been pursued to produce novel numerical algorithms intrinsically parallelizable. By the partition of the computational domain into subdomains the original problem is reformulated upon each subdomain, yielding a family of almost independent subproblems of lower computational complexity. This domain decomposition can encompass the use of different numerical schemes within different subdomains. In this way, the FEM-BEM coupling can be solved separately in the non linear FEM region and in the linear BEM region preserving the advantages of each method. Consistency of the subdomain problem with the original one is ensured by enforcing suitable transmission of information between adjacent subregions.

This paper presents a study about the different possibilities of performing the coupling in non linear problems. In the standard coupling, possible implementations orientated to eliminating the disadvantages of this type of coupling are studied in a detailed way. In addition to the standard coupling, we present an alternative which makes a parallel resolution possible. It is based in a partition of the original domain into subregions enforcing suitable transmission of information between adjacent subregions. Such an exchange of information is handled by proper interface operators whose numerical approximation is studied.

Numerical results are given which indicate the performance of the different proposed possibilities.

\section{Rate-Independent Plasticity.Continuum Formulation}

We summarize below the evolution equations for classical rate-independent plasticity. Attention is restricted to the static infinitesimal theory.

\subsection{Basic Equations}

We let $\Omega \subset \Re^{\text {ndim }}$, with $1 \leq n_{\text {dim }} \leq 3$, be the domain of an elastoplastic body, with smooth boundary $\partial \Omega$; we denote, following standard notation, by $\varepsilon, \varepsilon^{p}$ y $\varepsilon^{e}$ the total, plastic and elastic strain tensors and $\sigma$ the stress tensor. The strain hardening is assumed to be described by a $n_{\text {int }}$-dimensional vector $\mathrm{Q}: \Omega \rightarrow \mathfrak{R}^{\text {nint }}\left(\mathrm{n}_{\text {int }} \geq 1\right)$.

Using a compact notation we can refer

$$
\Sigma=(\sigma, \mathrm{Q})
$$

as the generalized stress constrained to be within the convex elastic domain 


$$
P=\{\Sigma=(\sigma, Q) / \phi(\Sigma) \leq 0\}
$$

The elastic response is characterized as

$$
\sigma=D^{e}:\left(\varepsilon-\varepsilon^{p}\right)
$$

where it is assumed that the elasticity tensor $D^{c}$ is constant.

The classical rate independent plasticity model [7] is obtained through the local optimality conditions associated with the principle of maximum plastic dissipation

$$
\begin{aligned}
& \dot{\boldsymbol{\varepsilon}}^{\mathbf{p}}=\gamma \frac{\partial \phi(\Sigma)}{\partial \sigma} \\
& \dot{\mathbf{q}}=-\mathrm{H}^{-1} \dot{\mathrm{Q}}=\gamma \frac{\partial \phi(\Sigma)}{\partial \mathrm{Q}}
\end{aligned}
$$

where $\gamma$ is a plastic consistency parameter, which satisfies the following Kuhn-Tucker complementary conditions

$$
\gamma \geq 0 \quad \phi(\Sigma) \leq 0 \quad \gamma \phi(\Sigma)=0
$$

In the equations above $H$ is $a n_{\text {int }} \times n_{\text {int }}$ given matrix of generalized hardening moduli which is assumed to be positive definite and constant.

The conventional solution of incremental plasticity problems is based on explicit integration of the following constitutive equations

$$
\dot{\sigma}=\mathrm{D}^{\mathrm{ep}}: \dot{\varepsilon}
$$

where $D^{\text {ep }}$ is the elastoplastic tangent moduli [2] given by the expression

$$
D^{\text {ep }}=D^{e}-\frac{\left(D^{e}: \frac{\partial \phi}{\partial \sigma}\right) \otimes\left(D^{e}: \frac{\partial \phi}{\partial \sigma}\right)}{\frac{\partial \phi}{\partial \sigma}: D^{e}: \frac{\partial \phi}{\partial \sigma}+\frac{\partial \phi}{\partial Q}: H: \frac{\partial \phi}{\partial Q}}
$$

\section{Rate-Independent Elastoplasticity. Discrete Formula- tion}

The evolution equations. of elastoplasticity define a constrained problem of evolution. From a computational standpoint it is essential to note that this problem of evolution may be regarded as strain driven.

\subsection{Closest-point Projection Algorithm}

At time $t_{n} \in[0, T]$ we assume that the total $\varepsilon_{n}$ and plastic $\varepsilon_{n}^{p}$ strain fields and the internal variables $q_{n}$ are known. Assuming that $\Delta \mathrm{u}$ is the incremental displacement field given, the basic problem is to update the fields $\sigma_{n+1}, \varepsilon_{n+1}^{p}$, $q_{n+1}$ to $t_{n+1} \in[0, T]$ in a manner consistent with the elastoplastic constitutive equations from the preceding paragraph.

By application of an implicit backward Euler difference scheme, the problem of evolution is transformed into the following constrained optimization problem

$$
\mathrm{E}_{\mathrm{n}+1}=\mathrm{E}_{\mathrm{n}}+\Delta \mathrm{E}
$$

$$
\begin{gathered}
\Sigma_{n+1}=T:\left(E_{n+1}-X_{n+1}\right) \\
X_{n+1}=X_{n}+\Delta \gamma \nabla \phi\left(\Sigma_{n+1}\right)
\end{gathered}
$$

governed by the discrete Kuhn-Tucker conditions:

$$
\begin{gathered}
\phi\left(\Sigma_{n+1}\right) \leq 0 \\
\Delta \gamma \geq 0 \\
\Delta \gamma \phi\left(\Sigma_{n+1}\right)=0
\end{gathered}
$$

where, for simplicity in the algorithmic analysis, we have introduced the compact notation

$$
E=(\varepsilon, 0) \quad X=\left(\varepsilon^{p}, q\right) \quad T=\operatorname{diag}\left[D^{e}, H\right]
$$

In order to interpret the algorithm geometrically, an additive decomposition $[8,9,10]$ of Equations $(9,10,11,12$, $13,14)$ is made. Introducing the following trial elastic state

$$
\begin{gathered}
\Sigma_{n+1}^{\text {test }}=T:\left(E_{n+1}-X_{n+1}^{\text {test }}\right)=\Sigma_{n}+T: \Delta E \\
X_{n+1}^{\text {test }}=X_{n} \\
\phi_{n+1}^{\text {test }}=\phi\left(\Sigma_{n+1}^{\text {test }}\right)
\end{gathered}
$$

the Equations $(9,10,11)$ may be interpreted assuming that all of the prescribed deformation is invested in elastically straining the material remaining the plastic strains and internal variables unchanged.

In a second plastic corrector state

$$
\begin{gathered}
\Sigma_{n+1}=\Sigma_{n+1}^{\text {test }}-T: X_{n+1} \\
X_{n+1}=X_{n+1}^{\text {test }}+\Delta \gamma \nabla \phi\left(\Sigma_{n+1}\right)
\end{gathered}
$$

the elastically predicted stresses are relaxed onto a suitably updated yield surface.

In a geometric interpretation, the final state $\left(\sigma_{n+1}, Q_{n+1}\right)$ is the closest point-projection in $\Sigma$-space of $\left(\sigma_{n+1}^{\text {test }}, Q_{n+1}^{\text {test }}\right)$ onto the boundary of the elastic region $P$. For an arbitrary convex yield function, the numerical integration of the elastoplastic problem is thus reduced to the minimization problem of finding the minimum distance [9] of a point to a convex set.

The algorithm can be exactly linearized in closed form. This leads to the notion of consistent [11] as opposed to continuum tangent elastoplastic moduli. This moduli is asymptotically consistent with the continuum moduli but it preserves the quadratic rate of asymptotic convergence.

The expression of the symmetric consistent tangent elastoplastic moduli [12] for the closest point algorithm is defined as

$$
\hat{D}=\left.\frac{d \sigma}{d \varepsilon}\right|_{n+1}=\left\langle\begin{array}{ll}
I & 0
\end{array}\right\rangle\left(A^{-1}-\frac{A^{-1}: N_{n+1} \otimes A^{-1}: N_{n+1}}{N_{n+1}^{t}: A^{-1}: N_{n+1}}\right)\left(\begin{array}{l}
I \\
0
\end{array}\right)
$$

where

$$
N_{n+1}^{t}=\left(\partial \phi / \partial \sigma_{n+1} \quad \partial \phi / \partial Q_{n+1}\right)
$$

and 


$$
A=\left(\begin{array}{cc}
D^{e-1}+\Delta \gamma \frac{\partial^{2} \phi}{\partial \sigma_{n+1}^{2}} & \Delta \gamma \frac{\partial^{2} \phi}{\partial \sigma_{n+1} \partial Q_{n+1}} \\
\Delta \gamma \frac{\partial^{2} \phi}{\partial Q_{n+1} \partial \sigma_{n+1}} & H^{-1}+\Delta \gamma \frac{\partial^{2} \phi}{\partial Q_{n+1}^{2}}
\end{array}\right)
$$

\section{Standard BEM-FEM Coupling}

As it is well-known, in the FEM the starting expression is the equation of virtual work

$$
\int_{\Omega} \sigma \delta \varepsilon d \Omega=\int_{\Omega} b \delta u d \Omega+\int_{\Gamma_{\sigma}} \bar{t} \delta u d \Gamma_{\sigma}
$$

From this equation, the standard finite element system can be written as

$$
\mathrm{Ku}=\mathrm{F}+\mathrm{D}
$$

where $K$ is the stiffness matrix for the system, $F$ the equivalent nodal force vector and $\mathrm{D}$ the vector due to the body force. We can write $F$ as

$$
\mathrm{F}=\mathrm{Nt}
$$

where $\mathrm{N}$ represents the Gram matrix and $\mathrm{t}$ is a vector of nodal tractions.

The starting expression for boundary element is

$$
c u+\int_{\Gamma} t^{*} u d \Gamma=\int_{\Gamma} u^{*} t d \Gamma+\int_{\Omega} b u^{*} d \Omega
$$

where $u^{*}$ represents the fundamental solution. This expression leads to the system [3]

$$
\mathrm{Hu}=\mathrm{Gt}+\mathrm{B}
$$

$\mathrm{H}$ and $\mathrm{G}$ being dependent on the fundamental tractions and displacements respectively and $B$ representing the vector due to body forces.

We consider a problem consisting of two domains $\Omega_{1}$ and $\Omega_{2}$ joined by an interface $\Gamma_{1}$, and which makes use of a finite element formulation in $\Omega_{l}$ and a boundary element formulation in $\Omega_{2}$. In order to join the two parts it is necessary to apply compatibility and equilibrium conditions along the interface $\Gamma_{I}$, i.e.

$$
\begin{aligned}
& u_{11}=u_{12} \\
& t_{11}=t_{12}
\end{aligned}
$$

Traditionally, we have two standard alternatives $[3,13$, 14] as to how to approach the problem. We may develop the boundary element region $\Omega_{2}$ as an equivalent finite element, assemble the effective stiffness matrix with those of finite elements of region $\Omega_{1}$ and solve the overall system as a stiffness problem. Alternatively we can consider $\Omega_{1}$ and $\Omega_{2}$ as if they were both boundary element regions.

Using the first approach we can transform Equation (28) by inverting $G$ and premultiplying by $N$, obtaining the following finite element form

$$
\mathbf{K}^{\prime} \mathbf{u}=\mathbf{F}^{\prime}+\mathbf{D}^{\prime}
$$

where

$$
K^{\prime}=N^{-1} H \quad D^{\prime}=N^{-1} B \quad F^{\prime}=N t
$$

With the second approach and according to Equations (29, 30) we have a system

$$
\left[\begin{array}{cccc}
K_{1} & K_{11} & N_{11} & 0 \\
0 & H_{12} & -G_{12} & H_{2}
\end{array}\right]\left[\begin{array}{l}
u_{1} \\
u_{1} \\
t_{1} \\
u_{2}
\end{array}\right\}=\left[\begin{array}{cc}
N_{1} & 0 \\
0 & G_{2}
\end{array}\right]\left\{\begin{array}{l}
t_{1} \\
t_{2}
\end{array}\right\}
$$

where the variables in the subdomains, $\Omega_{1}$ and $\Omega_{2}$, and in the interface $\Gamma_{\mathfrak{l}}$, are considered separately.

The one that appears more interesting is that for which the boundary element region is treated as a finite element, since it can be easily incorporated into existing finite element computer packages but, in general, depending on the problem the first or the second approach will be more interesting.

\subsection{Non-linear Problems}

In cases such as soil plasticity, a modelling of a physical system of infinite extent is needed. It is well known that the BEM yields an ideal approach for treating the infinite halfspace representing the soil, but it is not convenient in treating non-linear media.

By a coupling method combining boundary and finite elements the problem can be simulated suitably: the finite element method to represent the non linear behavior of the soil and the boundary elements to represent the linear infinite halfspace. So the best features of each method are exploited in the simulation.

In this case, the BEM region coincides with the infinite interface between both subregions while the non linear zone is massive. Since the non linear zone is predominant, the number of degrees of freedom is much bigger in the FEM region than in the BEM region. Because of this, in the coupling we use the first proposed approach, considering the boundary element region as an equivalent finite element.

Considering separately the variables belonging to $\Omega_{l}$ of the variables in the linear interface, coincident with $\Omega_{2}$ $\left(\Omega_{2} \equiv \Gamma_{1}\right)$ (Figure 1), the equilibrium equation in the FEM region is expressed as

$$
\left(\begin{array}{l}
F_{\text {int }}^{1} \\
F_{\text {int }}^{2}
\end{array}\right)=\left(\begin{array}{c}
F_{\text {ext }}^{1} \\
F_{\text {ext }}^{2}
\end{array}\right)
$$

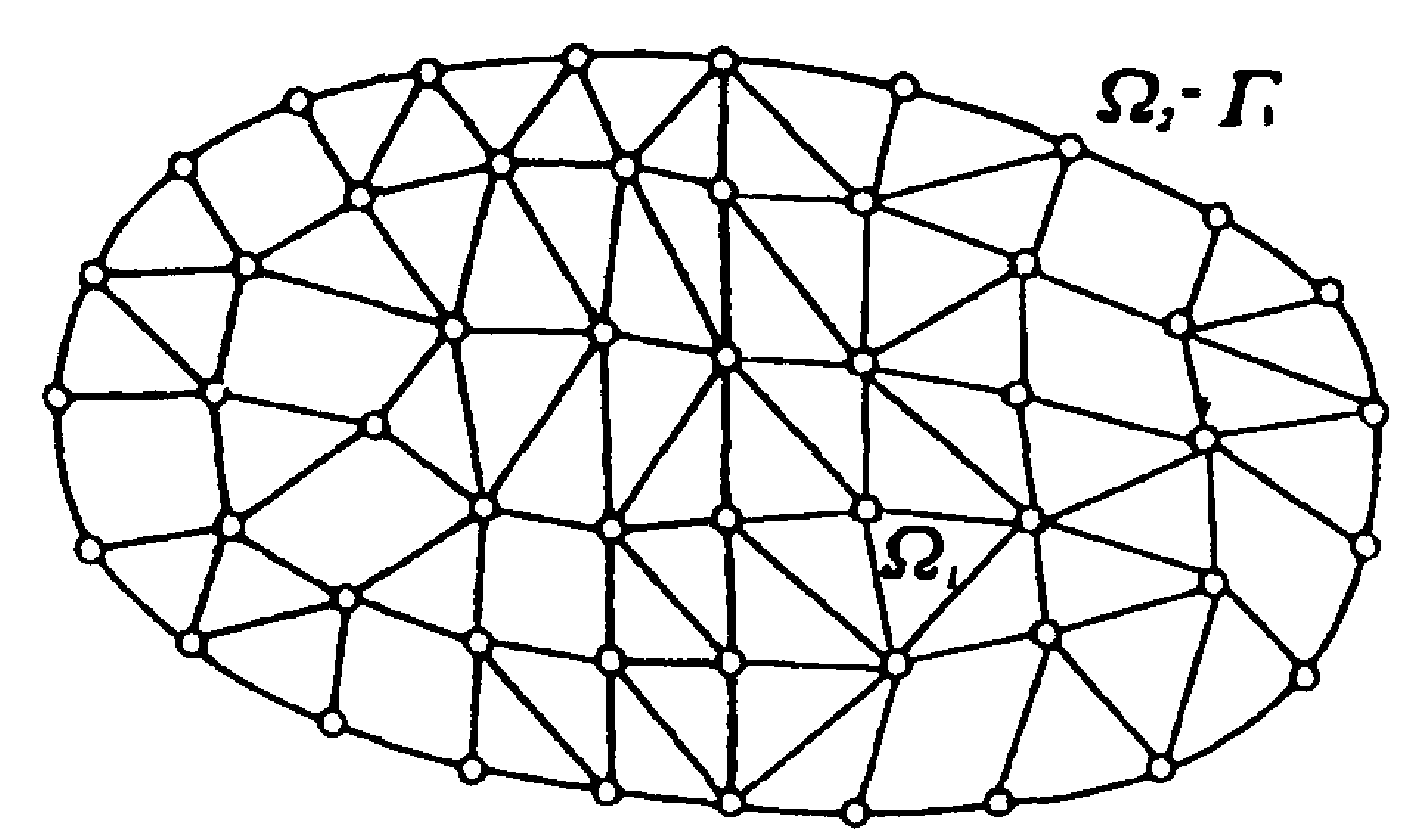

Figure 1: BEM-FEM coupling in an infinite halfplane 
The boundary element system of Equation (28), without considering the body forces, is formulated in the interface $\Omega_{2} \equiv \Gamma_{1}$ as

$$
\mathrm{NG}^{-1} \mathrm{Hu}_{2}=\mathrm{Nt}_{2}
$$

This is by no means a limitation of the method. Body forces or initial stresses derivable from a potential can be reduced to the boundary as shown, for instance, in Rizzo [15].

Adding Equations (34) and (35) taking into account the equilibrium condition (30) in the interface, the problem will be reduced to finding the displacements such that

$$
\begin{aligned}
& \mathrm{F}_{\text {int }}^{1}=\mathrm{F}_{\mathrm{ext}}^{\mathrm{l}} \\
& \mathrm{F}_{\text {int }}^{2}+\mathrm{Mu}_{2}=0
\end{aligned}
$$

where

$$
\mathrm{M}=\mathrm{NG}^{-1} \mathrm{H}
$$

The non linear system in $\Omega_{1}$ region can be solved iteratively with Newton's method. Linearizing the system about $\mathbf{u}^{k}$ yields

$$
\left(\begin{array}{cc}
A_{11} & A_{12} \\
A_{21} & A_{22}+M
\end{array}\right)^{k}\left(\begin{array}{c}
\Delta u_{1} \\
\Delta u_{2}
\end{array}\right)^{k}=\left(\begin{array}{c}
R_{1} \\
0
\end{array}\right)^{k}
$$

where

$$
\begin{array}{ll}
A_{11}=\left(F_{\text {int }}^{1}\right)_{u_{1}}^{\prime} & A_{12}=\left(F_{\text {int }}^{1}\right)_{u_{2}}^{\prime} \\
A_{21}=\left(F_{\text {int }}^{2}\right)_{u_{1}}^{\prime} & A_{22}=\left(F_{\text {int }}^{2}\right)_{u_{2}}^{\prime}
\end{array}
$$

and the residual

$$
R_{1}=F_{e x t}^{1}-F_{\text {int }}^{1}
$$

Solving Equation (38) can be done in two different ways taking into account the behavior of $\Omega_{2}$ region. The first approach is summarized in Figure 2. In this approach the linear behavior in $\Omega_{2}$ is preserved. If we note $n_{1}$ the number of non-null columns of $A_{21}, n_{2}$ the size of $A_{22}$ and $n_{3}$ the size of $A_{11}$, for each iteration we have to solve:
(i) $\left[A_{11}-A_{12}\left(A_{22}+M\right)^{-1} A_{21}\right]^{k} \Delta u_{1}^{k}=R_{1}^{k}$
(ii) $u_{1}^{k+1}=u_{1}^{k}+\Delta u_{1}^{k}$
(iii) $\mathrm{R}_{1}^{\mathrm{k}+1}=\left(\mathrm{F}_{\text {ext }}^{1}\right)-\left(\mathrm{F}_{\text {int }}^{1}\right)^{\mathrm{k}+1}$
(iv) if $\left\|\mathrm{R}_{1}^{\mathrm{k}+1}\right\|>\mathrm{TOL}$ then
$\mathrm{k} \leftarrow \mathrm{k}+1$
goto (i)
else
endif

$$
\text { goto }(v)
$$
(v) $\left(\mathrm{A}_{22}+\mathrm{M}\right) \Delta \mathrm{u}_{2}=-\mathrm{A}_{21} \Delta \mathrm{u}_{1}$

Figure 2: Standard coupling preserving the linearity of the interface
- $n_{1}$ non symmetric full systems of order $n_{2}$ to obtain $\left(A_{22}+M\right)^{-1} A_{21}$

-1 non symmetric full system of order $n_{3}$ to obtain $\Delta u_{1}^{k}$ and after the iterative process:

- 1 non symmetric full system of order $n_{2}$ to obtain $\Delta u_{2}$. All the systerns are non symmetric so the solving is very expensive. In this approach, the main disadvantage is eliminating the symmetry and the sparsity of $A_{11}$, obtained in the massive domain. However, when we solve, the linearity of the $\Omega_{2}$ region is preserved.

The second approach to solving Equation (38) would be orientated to preserving the symmetry and sparsity of $A_{11}$. To attain this we solve the $\Omega_{2}$ region as a non linear region, e.g. iteratively. This approach is summarized in Figure 3.

Figure 3: Standard coupling enforcing the non linearity in the interface

Noting $n_{1}$ the number of non-null columns of $A_{12}$ in each iteration we have to solve:

- $n_{1}$ sparse symmetric systems of order $n_{2}$ to obtain $A_{11}^{-1} A_{12}$

- 1 sparse symmetric system of order $n_{2}$ to obtain $A_{11}^{-1} R_{1}$

-1 full non symmetric system to obtain $\Delta u_{2}^{k}$

- 1 sparse symmetric system to obtain $\Delta u_{1}^{k}$

The main advantage of this approach is the possibility of solving iteratively the symmetric problems (non linear problems in the massive domain) by a preconditioned conjugate gradient method. The fact that several systems have to be solved iteratively is not too time consuming because $A_{12}$ is generally sparse and the preconditioner is performed only once. However, the main disadvantage consists of using the Newton-Raphson iterations to solve a linear region $\Omega_{2}$.

Of the two possibilities (Figure 2 and Figure 3), the second approach produces in the massive region $\Omega_{1}$ symmetric and sparse systems which decreases the cost of the resolution. For this reason, we use this second approach to obtain results when a standard coupling is performed. 


\section{Interface Relaxation Procedure}

Another approach to performing the BEM-FEM coupling is based on the decomposition of the initial problem into two subdomains. By this procedure, we solve the differential equations in separate mesh resolutions and iterate between subdomains until convergence is reached at the BEM-FEM interface $[16,17]$. It yields a family of almost independent subproblems of lower computational complexity.

This approach allows for the separate treatment of the linear and non linear problems keeping the advantages of both the BEM and FEM methods.

Consistency of the subdomain problem with the original one is ensured by enforcing suitable transmission of information between adjacent subregions using compatibility and equilibrium. To achieve convergence, at each iteration, a relaxation is accomplished at the subdomain interface.

For our problem, the linear BEM domain agrees with the interface between subdomains while the FEM domain represents a non linear behavior. From the original problem

$$
\operatorname{div} \sigma+f=0 \quad \text { in } \Omega
$$

with the boundary conditions

$$
\begin{array}{ll}
\mathbf{u}=\overline{\mathbf{u}} & \text { in } \Gamma_{\mathrm{D}} \\
\sigma \mathrm{n}=\overline{\mathbf{t}} & \text { in } \Gamma_{\mathrm{N}}
\end{array}
$$

the partitioned problem can be performed by the following algorithm [18]:

"Denoting by $u^{(1)}$ and $u^{(1)}$ the restrictions of $u$ to $\Omega_{1}$ and $\Gamma_{I}$ respectively and being $u^{(1) k}$ and $u^{(1) k}$ available for $k \geq 0$, then compute $u^{(1) k+1}$ and $u^{(1) k+1}$ by solving

$$
\begin{array}{r}
\operatorname{div} \sigma^{(I) k+1}+f=0 \quad \text { in } \quad \Gamma_{I} \\
u^{(I) k+1}=\theta u^{(1) k}+(1-\theta) u^{(I) k} \quad \text { in } \quad \Gamma_{I} .
\end{array}
$$

and

$$
\begin{array}{ccc}
\operatorname{div} \sigma^{(1) k+1}+\mathrm{f}=0 & \text { in } & \Omega_{1} \\
u^{(1)}=\mathrm{u} & \text { in } & \Gamma_{D} \\
\sigma^{(1)} \mathrm{n}=\mathrm{t} & \text { in } & \Gamma_{\mathrm{N}} \\
\mathrm{t}^{(1) \mathrm{k}+1}=\mathrm{t}^{(1) \mathrm{k}+1} & \text { in } \quad \Gamma_{I}
\end{array}
$$

and $0<\theta<1$ is a relaxation parameter".

In this approach a Dirichlet problem in the interface is solved by the BEM method and a Neumann problem in the interface is solved in the non linear FEM subdomain (Dirichlet-Neumann method). The tractions obtained with the BEM method are used as boundary conditions in the FEM region. The more accurate tractions obtained with the BEM method are determinant for the choice of the non linear region as a Neumann region in the interface. On the other hand, the Dirichlet boundary conditions in the BEM domain are obtained through a relaxation at the interface.

The relaxation parameter $\theta \in] 0,1[$ can be constant or selected dynamically [19] by means of a simple formula in order to maximize the rate of convergence of the above iteration by subdomain procedure. For this, let $z^{k}(\theta)$ be the sequence of functions corresponding to the relaxation procedure

$$
z^{k}(\theta)=\theta u^{(1) k}+(1-\theta) u^{(I) k}
$$

We take $\theta=\theta^{k}$ such that it is the number minimizing $\left\|z^{k}(\theta)-z^{k-1}(\theta)\right\|^{2}$. We obtain

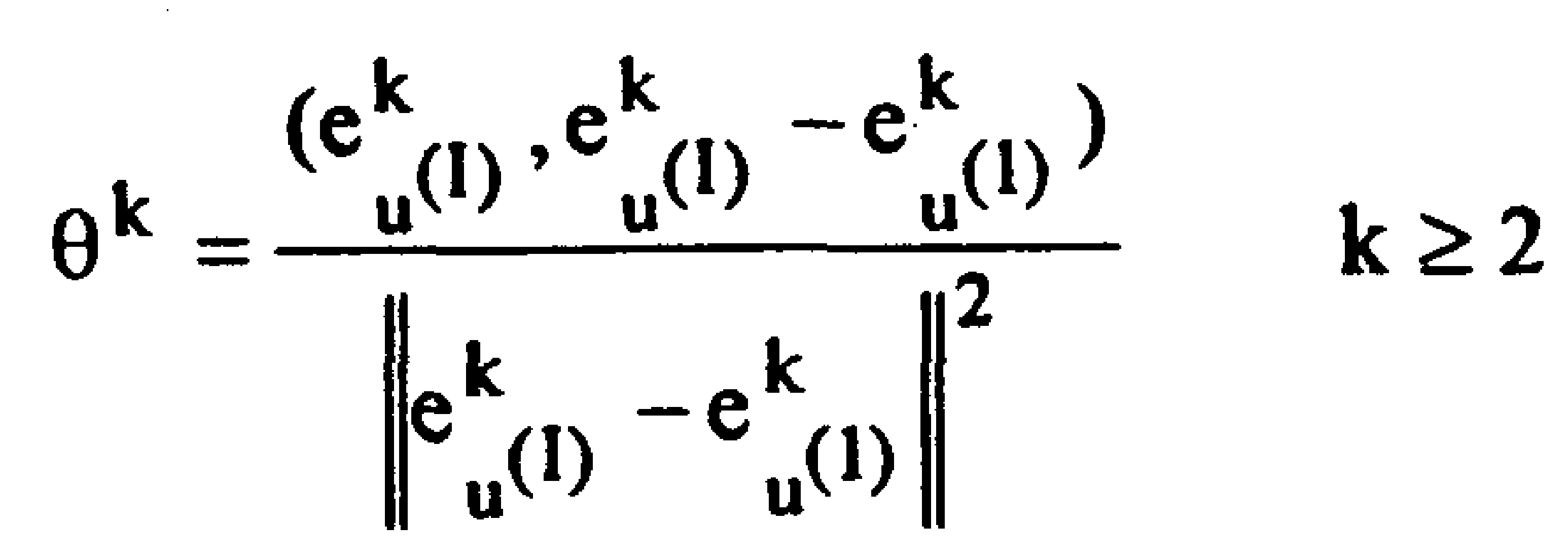

where

$$
\begin{aligned}
& e_{u_{(1)}^{k}}^{k} u^{(1) k}-u^{(1) k-1} \\
& e_{u}^{k}(1)=u^{(1) k}-u^{(1) k-1}
\end{aligned}
$$

while $\theta^{1}$ shouid be prescribed.

Being the Steklov-Poincare operator $S_{i}[20,21]$ (or the Schur complement matrix) given by

$$
S_{i} u^{(I)}=\left.\frac{\partial u_{i}\left(u^{(I)}, 0\right)}{\partial n_{i}}\right|_{\Gamma_{1}}
$$

and the right-hand side $b$

$$
b=-\frac{\partial \mathrm{u}_{1}(0, \mathrm{f})}{\partial \mathrm{n}_{1}}-\frac{\partial \mathrm{u}_{2}(0, \mathrm{f})}{\partial \mathrm{n}_{2}}
$$

the equilibrium condition (30) in the interface becomes

$$
\left(S_{1}+S_{2}\right) u^{(1)}=b
$$

From the concept of the Steklov-Poincare operator, the iteration-by-subdomain method can be interpreted in different ways: as a succesive under relaxation method in the interface corresponding to the splitting $S=S_{2}-\left(-S_{1}\right)$ with relaxation matrix

$$
L=S_{2}^{-1}\left[(1-\theta) S_{2}-\theta S_{1}\right]
$$

or, as a conjugate gradient method for the system (55) preconditioned by the matrix $S_{2}$.

\section{Preconditioning}

Newton's method is very attractive for solving a system of non linear equations, but it may be extremely expensive because it requires the solution of a linear system at each iteration step. An alternative way of improving the efficiency of Newton's method when it is used in large systems is by a composite Newton-Preconditioned Conjugate Gradient (PCG) technique [22] for the linearized system in lieu of the conventional LDU factorization of the global tangent operator.

If the system to solve is like $\mathrm{Ku}=\mathrm{F}$, a good preconditioner $\mathrm{C}$ is one such that the equivalent system $\mathrm{C}^{-1} \mathrm{Ku}=\mathrm{C}^{-1} \mathrm{~F}$ is easy 
to solve and the condition number of the iterative matrix is close to the unity. The only restriction to the choice of the preconditioning matrix $\mathrm{C}$ is provided by the operational cost and the preservation of the symmetric positive definite form of matrix $\mathrm{K}$.

According to these criteria, we should use a matrix easy to factorize and which is a good approximation of $K$ to increase the convergence. As a preconditioner we use the tangent operator $[23] K_{T 0}=-\left[\left(F_{i n t}^{\prime}\right)_{u_{1}}^{\prime}\right]^{1}$ during the first iteration of every load step. This preconditioner has the typical advantages and disadvantages of the Newton's method; $\mathrm{K}_{\mathrm{TO}}$ contains information on the present value of the load step so that the condition number of the matrix product $\mathrm{C}^{-1} \mathrm{~K}_{\mathrm{T}}$ is close to the unity; the method would be direct during the first iteration due to the necessary factorization of C.

\section{Numerical Results}

In this section we compare the performance of each of these coupling algorithms. The code used in the present study is an enhanced non linear version of DLEARN (Hughes 1987) [24] in which we have incorporated the boundary elements. All computations were performed in double precision on a SPARC station 2 supercomputer. As a constitutive model to simulate the deformation behavior of the soil the DruckerPrager elastoplastic model was used.

Convergence of the non linear algorithm is measured in terms of the residual norm which is given by

$$
\left\|\mathrm{R}^{k}\right\| \leq \varepsilon_{\mathrm{R}}\left\|\mathrm{R}^{0}\right\|
$$

where error tolerance is set at $\varepsilon_{\mathrm{R}}=10^{-3}$. The error tolerance for the PCG secondary iterations is $10^{-2}$. Material responses are sampled at the Gauss points using a nine-point integration in two-dimensional quadrilateral elements.

\subsection{Plane Strain Example}

As an example we consider the effects of initial stresses on a perforation with a horseshoe-like geometry at a depth of $60 \mathrm{~m}$. with an inner radius of $5 \mathrm{~m}$. The infinite halfplane modelized by boundary elements is located to three times the radius. Two-dimensional quadratic finite elements and one dimensional quadratic boundary elements are employed. For simplicity, we consider a homogeneous soil with the following constants: Young's modulus $E=2.5106 \mathrm{t} / \mathrm{m}^{2}$, Poisson's ratio $v=0.27$ and density $\gamma=2.4 \quad \mathrm{t} / \mathrm{m}^{3}$; the unconfined compression strength $R_{c}$ is $2240 \mathrm{t} / \mathrm{m}^{2}$ and the angle of internal friction $\phi=33^{\circ}$. Initial stresses of the soil were generated internally at each integration point and after an internal pressure was used to proceed with the computations.

It is interesting to study the performance of the coupling algorithm when the mesh is refined progressively. For this reason, three different meshes are employed in the calculations (Figure 4). These three meshes have 504, 864 and 1320 degrees of freedom respectively.
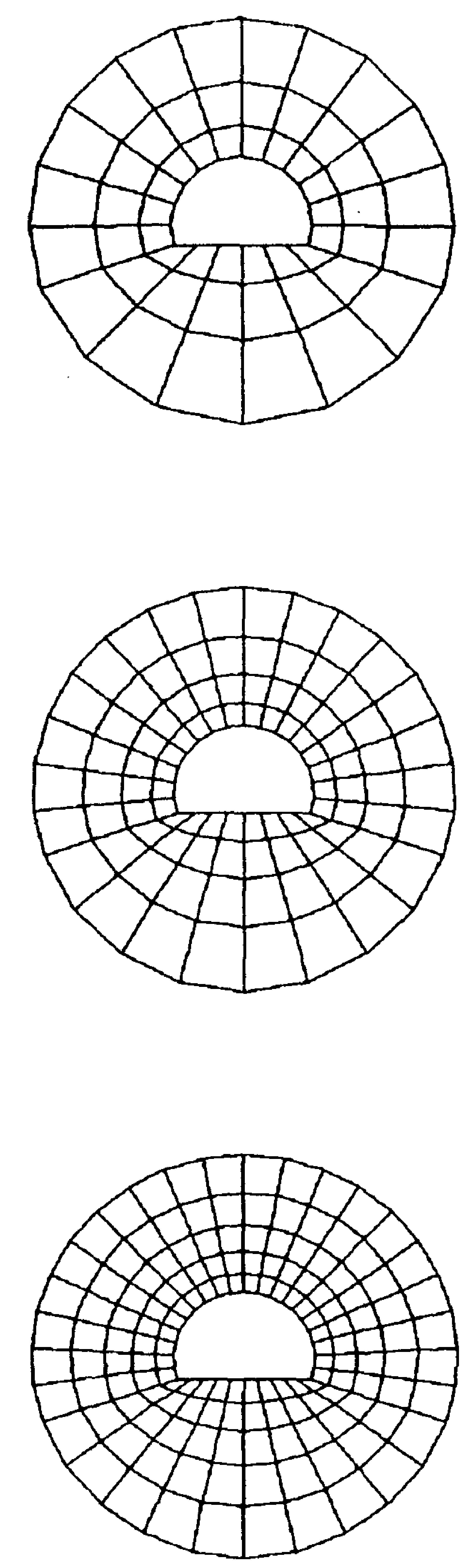

Figure 4: Different meshes used in the calculations

The calculations reported here were performed in four different load steps and four alternative algorithms were employed:

a. Traditional coupling algorithm (Figure 3 ) with the full Newton method employing Crout triangular factorization for direct equation solving (TRAD-NR-D)

b. Traditional coupling algorithm (Figure 3) with the composite Newton-PCG iteration (TRAD-NR-PCG)

c. Coupling by the Dirichlet-Neumann relaxation procedure with the full Newton method employing Crout triangular factorization for direct equation solving. The relaxation parameter is selected dynamically (RELAJ-NR-D)

d. Coupling by the relaxation procedure with the composite Newton-PCG iteration with a dynamic relaxation parameter (RELAJ-NR-PCG)

Tables 1,2 and 3 summarize the performance of each of the four algorithms for the three different meshes. When a relaxation procedure is used, we prefix three relaxation iterations in each load step. 


\begin{tabular}{|c|c|c|c|c|}
\hline Criteria & TRAD-NR-D & TRAD-NR-PCG & RELAJ-NR-D & RELAJ-NR-PCG \\
\hline No. load steps & 4 & 4 & 4 & 4 \\
\hline No. global iterations & 18 & 18 & 49 & 50 \\
\hline No. relaxat. iterations & - & - & 12 & 12 \\
\hline No. global iter/step & 4.5 & 4.5 & 12.25 & 12.5 \\
\hline No. global iter/relax.ite & - & - & 4.08 & 4.17 \\
\hline No. factorizations. & 18 & 4 & 49 & 12 \\
\hline No. subiterations & - & 1024 & - & 146 \\
\hline No.subiter./global iter. & - & 56.89 & - & 2.92 \\
\hline Total CPU(sec) & 390 & 639.004 & 369.020 & 312.027 \\
\hline
\end{tabular}

Table 1: Mesh 1- 504 dof

\begin{tabular}{ccccc} 
Criteria & TRAD-NR-D & TRAD-NR-PCG & RELAJ-NR-D & $\begin{array}{c}\text { RELAJ-NR- } \\
\text { PCG }\end{array}$ \\
\hline No. load steps & 4 & 4 & 4 & 4 \\
No.global iterations & 21 & 21 & 52 & 51 \\
No.relaxat.iterations & - & - & 12 & 12 \\
No.global iter./step & 5.25 & 5.25 & 13 & 12.75 \\
No.global iter./relax.iter & - & - & 4.33 & 4.25 \\
No.factorizations & 21 & 4 & 52 & 12 \\
No.subiterations & - & 1451 & - & 186 \\
No.subiter./global iter. & - & 69.09 & - & 3.65 \\
Total CPU(sec.) & 1232.05 & 1749.95 & 929.969 & 690.031 \\
\end{tabular}

Table 2: Mesh 2- 864 dof

$\begin{array}{cccccc}\text { Criteria } & \text { TRAD-NR-D } & \text { TRAD-NR-PCG } & \text { RELAJ-NR-D } & \text { RELAJ-NR-PCG } \\ \text { No.load steps } & 4 & 4 & 4 & 4 \\ \text { No.global iterations } & 19 & 20 & 50 & 50 \\ \text { No.relax.iterations } & - & -. .12 & 12 & 12 \\ \text { No.global iter./step } & 4.75 & 5 & 12.5 & 12.5 \\ \text { No.global iter./relax.iter. } & - & - & 4.17 & 4.17 \\ \text { No.factorizations } & 19 & 4 & 50 & 12 \\ \text { No.subiterations } & - & 1641 & - & 174 \\ \text { No.subiter./global.iter. } & - & 82.05 & - & 3.48 \\ \text { Total CPU(sec.) } & 2882.05 & 4154.98 & 1881.06 & 1325.98\end{array}$

Table 3: Mesh 3- 1320 dof

The results show that the relaxation procedures require less CPU compared with the standard coupling methods . This is more evident when the number of degrees of freedom increase; in spite of the number of global iterations being much higher in the relaxation methods than in the standard coupling, in the second case it is necessary to solve the system $A_{11}^{-1} A_{12}$ in each Newton's iteration; the number of subsystems to solve in $A_{11}^{-1} A_{12}$ increase progressively with the number of non null columns in $A_{12}$ and hence with the degrees of freedom; the performance is more inefficient when we use a standard coupling with PCG since the number of subiterations is very high due to the system $A_{11}^{-1} A_{12}$. Because of this, the TRAD-NR-PCG is not optimal to perform the coupling.

As for the relaxation method, the PCG is more optimal than Crout's factorization since it is only necessary to solve the system $A_{11} \Delta u_{1}=R_{1}$ in each Newton's iteration and the number of Crout's factorizations is less when the PCG is used. So the RELAJ-NR-PCG is the least expensive of all the proposed coupling possibilities.

\subsection{Relaxation Parameter}

In all the calculations we have adopted a dynamic relaxation parameter chosen according to Equation (51). In Table 4 we summarize the results obtained when a constant parameter is used during all the process in lieu of a dynamic parameter. It can be observed that $\theta$-values less than 0.5 are suitable while higher values are slower and even the algorithm can diverge.

We have predetermined three relaxation iterations in each load step. This conclusion is obtained from previous tests. If we denote by ERROR the natural logarithm of the 


\begin{tabular}{ccccccc} 
& $\theta$ DYNAMIC & $\theta=0.1$ & $\theta=0.3$ & $\theta=0.5$ & $\theta=0.7$ \\
\hline No.load steps & 4 & 4 & 4 & 4 & divergence \\
No.global iterations & 52 & 46 & 48 & 73 & divergence \\
No.global iter./step & 13 & 11.5 & 12 & 18.25 & divergence \\
Total CPU(sec.) & 323.97 & 286.84 & 299.32 & 408.498 & divergence
\end{tabular}

Table 4: Performance of the relaxation algorithm for different values of $\theta$

maximum among the errors at the interfaces between the $B E M$ solution and the FEM solution in the same relaxation iteration, we can represent ERROR with the number of relaxation iterations. For this, we have chosen a constant value $\theta=0.3$ (Figure 5) and a $\theta$-dynamic (Figure 6).

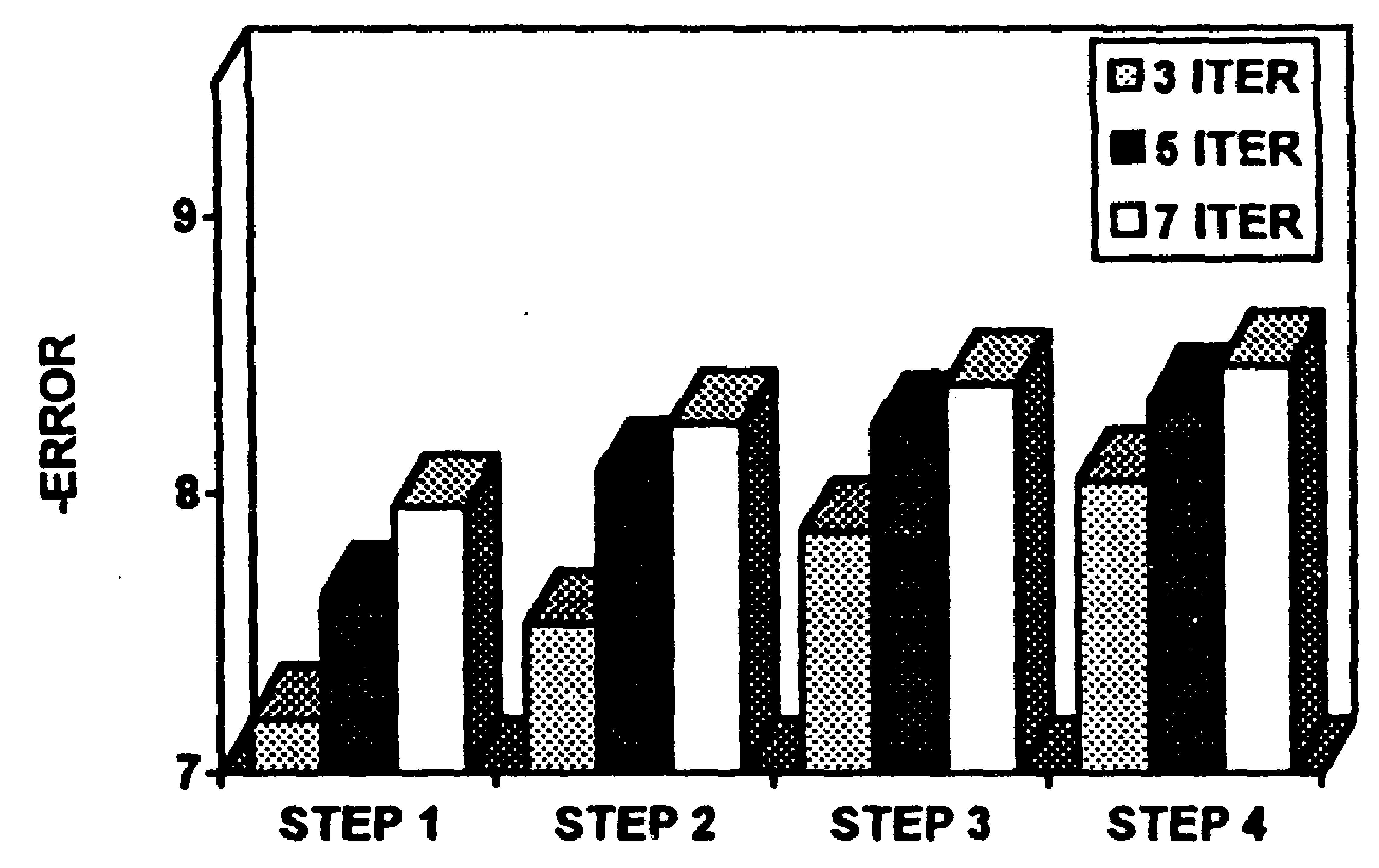

Figure 5: Evolution of ERROR with the number of relaxation iterations $(\theta=0.3)$

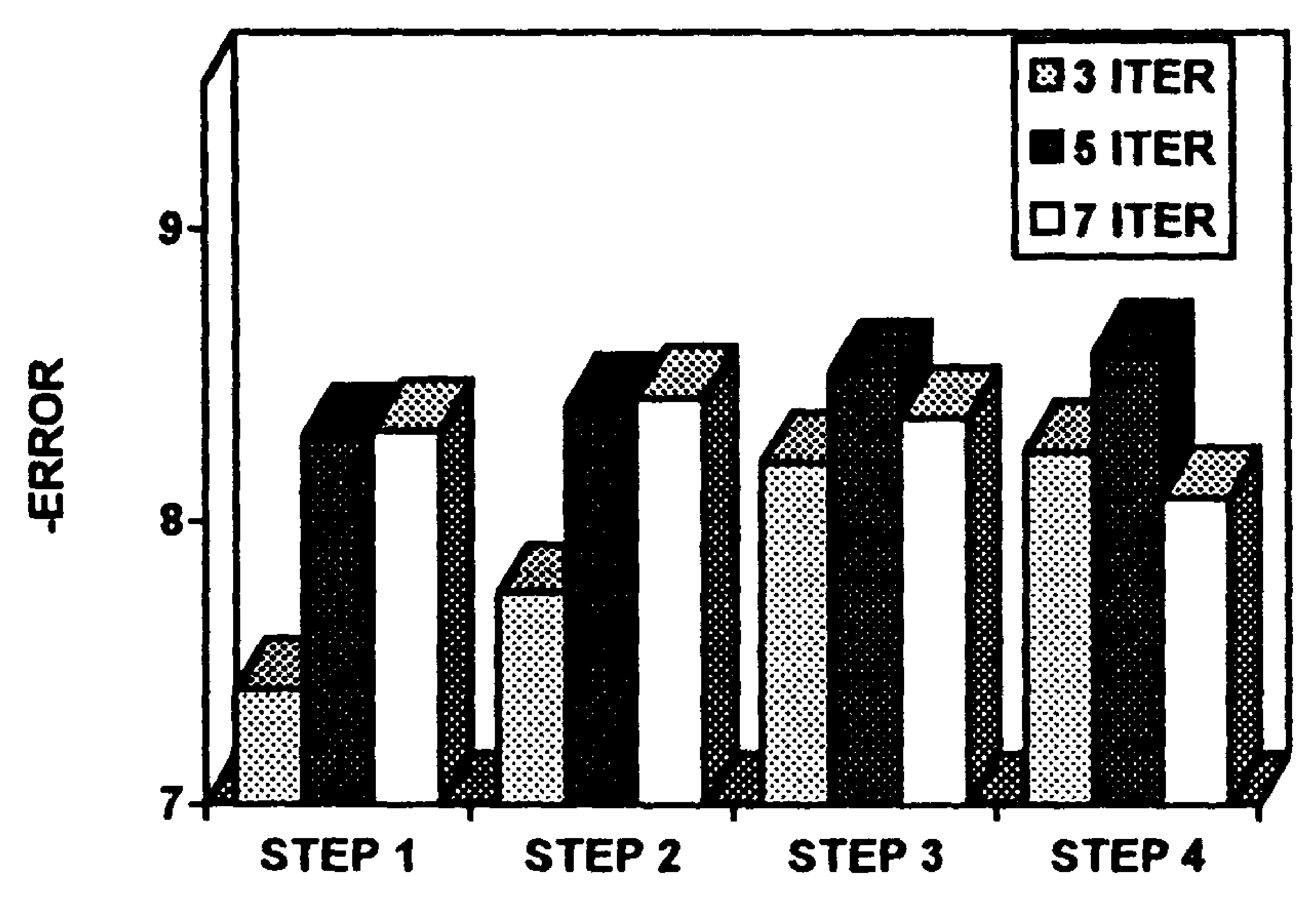

Figure 6: Evolution of ERROR with the number of relaxation iterations ( $\theta$-dynamic)

From the observation of Figure 6 we can deduce that a higher number of iterations does not involve a better accuracy, when a $\theta$-dynamic is employed. However, when we use a constant parameter (Figure 5) the accuracy increases with the number of iterations but, probably, this better accuracy does not compensate the higher cost.

\section{Conclusions}

Different FEM-BEM coupling algorithms have been investigated in a non linear context over an infinite halfplane. In addition to the traditionally used ways we have proposed a different alternative based on solving the BEM and FEM domains separately. This allows us to decouple the non linear and linear equations and solve the subproblems independently therefore allowing parallel computations within any multiprocessor environment.

Refering to the first approach (standard coupling), from a computational point of view, the enforcement of the linear halfplane as a non linear domain has turned out to be more efficient since it allows for the preservation of the symmetry and sparsity of the FEM matrix. The second approach, relaxation procedure, has resulted more optimal in CPU time. This would be more evident if a multiprocessor computer had been used.

The choice of the $\theta$ relaxation parameter has been important to guarantee the convergence of the method. Values less than $\mathbf{0 . 5}$ or dynamic have been necessary for this reason.

The comparison between the conventional LDU factorization of the global tangent operator and the iterative methods based on preconditioned conjugate gradients (PCG) for solving large systems of equations associated with a linearized problem has been developed. We have shown that the composite Newton-PCG technique possesses considerable potential for usefulness in large scale computations.

\section{References}

[1] Zienkiewicz, O.C., Kelly, D.W., Bettes, P., “ The coupling of the finite element and boundary solution procedures", in "Int. J. Num. Meth. Eng.", 11, 355-375, 1977.

[2] Zienkiewicz, O.C., Taylor, R.L., "The Finite Element Method", Mc Graw-Hill, London, 1991.

[3] Brebbia, C.A., Dominguez, J., "Boundary Elements:An Introductory Course", Computational Mechanics Publications, McGraw-Hill, 1989.

[4] Dryja, M., Widlund, O.B., "Towards an unified theory on domain decomposition algorithms for elliptic problems", in "Domain Decomposition Methods for Partial Differential Equations", Vol. 3, SIAM, Philadelphia, 321, 1990.

[5] Lions, P.L., " On the Schwarz alternating method I", in "Domain Decomposition Methods for Partial Differential Equations", SIAM, Philadelphia, 1988.

[6] Lions, P.L., " On the Schwarz alternating method II", in "Domain Decomposition Methods for Partial Differential 
Equations II", SIAM, Philadelphia, 1989.

[7] Owen D.R., Hinton, E., "Finite Elements in Plasticity:Theory and Practice", McGraw-Hill, New York, 1980.

[8] Hughes, T.J.R., "Numerical implementation of constitutive models: rate-independent deviatoric plasticity", in "Workshop on theoretical foundations for large scale computations in nonlinear material behavior", Northwestern University, Evanston, Illinois, 1983.

[9] Ortiz, M., Simo, J.C., "An analysis of a new class of integration algorithms for elastoplastic constitutive relations", in "Int. J. Numer. Meth. Eng.", 23, 353-366, 1986.

[10]Wilkins, M.L., "Calculation of elastoplastic-flow", in "Methods of Computational Physics", 3, Academic Press, New York, 1964.

[11]Simo, J.C., Taylor, R.L., "Consistent tangent operators for rate-independent elastoplasticity", in "Computer Methods in Applied Mechanics and Engineering" , 48 , 101-118, 1985.

[12]Ortiz, M., Popov, E.P., "Accuracy and stability of integration algorithms for elastoplastic constitutive relations", in "Int. J. Numer. Meth. Eng.",. 21, 1561$1576,1985$.

[13]Hartmann, F., "Introduction to Boundary Elements", Springer-Verlag, 1989.

[14]Margulies, M., "Combination of the boundary element and finite element methods", in "Progress in Boundary Element Methods", Pentech Press, London, 258-288, 1981.

[15]Rizzo, F.J., Shippy, D.J., "The boundary element method in thermoelasticity", in "Developments in Boundary Element Methods-1", Applied Science Publishers, 155-172 1979.
[16]Perera, R., Ruiz, A., Alarcon, E., “A FEM-BEM coupling procedure through the Steklov-Poincarè operator", in "Boundary Elements XV", 2, 621-632, 1993.

[17]Perera, R., “Acoplamiento MEF-MEC. Aplicación a elastoplasticidad", Ph.D.Thesis (in Spanish), Polytechnical University, Madrid, Spain, 1995.

[18]Marini, L.D., Quarteroni, A.,."An iterative procedure for domain decomposition methods: a finite element approach", in "Domain Decomposition Methods for Partial Differential Equations", Vol. 1, SIAM, Philadelphia, 129-143, 1988.

[19]Funaro, D., Quarteroni, A., Zanolli, P., "An iterative procedure with interface relaxation for domain decomposition methods", in "Siam J. Numer. Anal.", 6, 1213-1236, 1988.

[20]Bjorstad, P.E., Widlund, O.B., "Iterative methods for the solution of elliptic problems on regions partitioned into substructures", in "Siam J. Numer. Anal.",Vol. 23, 6, 1097-11201986.

[21]Agoshkov, V.I., "Poincaré-Steklov's operators and domain decomposition methods in finite dimensional spaces", in "Domain Decomposition Methods for Partial Differential Equations", Vol.1, SIAM, Philadephia, 73$112,1988$.

[22]Papadrakakis, M., Gantes, C.J., "Truncated Newton methods for nonlinear finite element analysis", in "Computers \& Structures", 30, 705-714, 1988.

[23]Borja, R.I., "Composite Newton-PCG and Quasi-Newton iterations for nonlinear consolidation", in "Computer Methods in Applied Mechanics and Engineering", 86, 27-60, 1991

[24]Hughes, T.J.R., "The Finite Element Method", PrenticeHall, New Jersey 1987. 